\title{
Pion Form Factor in Improved Holographic QCD Backgrounds
}

\author{
Herry J. Kwee* and Richard F. Lebed \\ Department of Physics, Arizona State University, Tempe, AZ 85287-1504
}

(Dated: December 2007)

\begin{abstract}
We extend a recent numerical calculation of the pion electromagnetic form factor $F_{\pi}\left(Q^{2}\right)$ in holographic QCD to study two important issues regarding the behavior of fields in the bulk. First, we show that using a chiral symmetry-breaking field formally satisfying the boundary conditions of the "soft-wall" model changes numerical results very little from the earlier calculation that ignores these constraints. Second, we use a background field that interpolates between "hard-wall" and "soft-wall" models to obtain an improved model that reproduces the desirable phenomenological features of both. In all cases, $F_{\pi}$ for large $Q^{2}$ is shallower than data, an effect that can be cured by relaxing the fit to one of the static observables, particularly the decay constant $f_{\pi}$.

PACS numbers: 11.25.Tq, 11.25.Wx, 13.40.Gp
\end{abstract}

${ }^{*}$ Electronic address: Herry.Kwee@asu.edu

${ }^{\dagger}$ Electronic address: Richard.Lebed@asu.edu 


\section{INTRODUCTION}

One of the most interesting developments in QCD in recent years has been the application to hadronic physics of the gauge/gravity correspondence [1] between strongly-coupled gauge theories and weakly-coupled gravity on curved spacetime backgrounds. This correspondence is most firmly established between $\mathcal{N}=4$ supersymmetric Yang-Mills theories (which are conformal field theories $[\mathrm{CFT}]$ ) and a 5-dimensional anti-de Sitter (AdS) gravity background - hence the moniker AdS/CFT. However, one may conjecture that a similar connection holds for any strongly-coupled gauge theory possessing an approximate conformal symmetry, a property that holds for QCD in its high-energy limit. The terms "AdS/QCD" or "holographic QCD" refer to studies based upon the premise that QCD belongs to this class and possesses a suitable gravity dual in 5D space. Properties such as confinement and chiral symmetry breaking arise in many models built in this way; indeed, in the "topdown" approach one starts with a string theory and chooses a gravity background that reproduces such basic QCD features. Much more amenable to phenomenological analysis is the "bottom-up" approach, in which one begins with the QCD observables and determines what gravity backgrounds reproduce them most successfully.

The fields of our 4D universe occupy only one surface of the 5D AdS space, whose metric is given by

$$
d s^{2}=g_{M N} d x^{M} d x^{N}=\frac{1}{z^{2}}\left(\eta_{\mu \nu} d x^{\mu} d x^{\nu}-d z^{2}\right) .
$$

Here the full nontrivial 5D metric $g_{M N}$ obtained from Eq. (1) is distinguished from the 4D Minkowski metric $\eta_{\mu \nu}=\operatorname{diag}(+,-,-,-)$. The ultraviolet (UV) limit of QCD is represented by fields living near the AdS singularity $z=\epsilon \rightarrow 0$ (the "UV brane"), suggesting the association of the extra "bulk" coordinate $z$ with inverse momentum scales: $Q \sim 1 / z$. The gauge/gravity correspondence states that every CFT operator $\mathcal{O}(x)$ is associated with a bulk field $\Psi(x, z)$ of given quantum numbers uniquely determined by its value $\Psi(x, \epsilon)$ on the UV brane, which explains the origin of the usage "holographic". Additionally, the global QCD symmetry of isospin associated with the two light quark flavors is promoted to a gauged $\mathrm{SU}(2)$ symmetry respected by the bulk fields.

From the point of view of hadronic physics, the original $\mathcal{N}=4$ super-Yang Mills theory is inadequate due to being exactly conformal and therefore lacking asymptotic $S$-matrix particle states. In AdS/QCD one breaks the conformal symmetry (and introduces a mass 
scale) by impeding the ability of the fields $\Psi(x, z)$ to penetrate deeply into the bulk, leading to an explanation of confinement and more generally constraining the model's infrared (IR) behavior. The most straightforward realization uses a hard cutoff at a particular value $z=z_{0} \sim 1 / \Lambda_{\mathrm{QCD}}$ (the "IR brane") at which point appropriate boundary conditions are imposed upon $\Psi(x, z)$, thus defining the so-called "hard-wall" model [2]. However, despite its economy, the hard-wall model leads to results incompatible with the expectations of linear confinement: For any model in which $\Psi(x, z)$ penetrates to a limited depth into the bulk, the eigenmodes of $\Psi$ (with mass eigenvalues $m_{n}$ ) represent hadronic states all carrying the same quantum numbers, producing the AdS/QCD version of Regge trajectories. However, QCD with linear confinement is expected [3] to follow the trajectory $m_{n}^{2} \sim n$, while hard-wall models predict $m_{n}^{2} \sim n^{2}$. To repair this shortcoming requires the introduction of a "soft-wall" gravity background with an exponential decrease $\sim e^{-\kappa^{2} z^{2}}$ in the action for large $z$ [4], in which case $\kappa$ serves the role of $\Lambda_{\mathrm{QCD}}$.

Studies of hadronic properties in AdS/QCD have been very popular in recent years; to name but a few that share the same spirit as this work are examinations of hadronic spectra [4, 5, 6, 7, 8, 9, 10, 11], the couplings of hadrons in the presence of chiral symmetry breaking [5, 12, 13, 14, 15], the quark-quark potential [16], 4-quark operators [17], and hadronic form factors [18, 19, 20, 21, 22, 23]. In this paper we continue a study of the pion electromagnetic form factor $F_{\pi}$ initiated by the present authors in Ref. [24], using the treatment of chiral symmetry-breaking effects (parametrized by a light quark mass $m_{q}$ and chiral condensate $\sigma$ ) explicitly incorporated into the Lagrangian, in the manner developed by the model of Ref. [5]. $F_{\pi}$ has also been considered in two other recent papers [22, 23]; Ref. 22. uses a model that does not incorporate chiral symmetry breaking explicitly, while Ref. [23] also uses the formalism of Ref. [5] and has a considerable overlap [37] with the hard-wall calculations of Ref. [24]; indeed, it also proves a number of analytical results that hold in the limit $m_{q}=0$. However, Ref. [23] does not present calculations in the soft-wall model due to objections that we address below.

While results for most hadronic quantities tend to agree surprisingly well with the results of the hard-wall model (and generally somewhat less well for the soft-wall model [19, 24]), observables in the vector meson sector tend to depend somewhat less sensitively on the precise nature of the IR boundary condition. However, the axial-vector sector and especially the pion depend upon chiral symmetry-breaking in a much more direct fashion [4]; exploring 
this sensitivity was an original motivation of the studies in Refs. [23, 24].

Our previous work [24] numerically calculated $F_{\pi}\left(Q^{2}\right)$ for spacelike values $Q^{2} \geq 0$ of momentum transfer, as well as couplings $g_{\rho^{(n)} \pi \pi}$ obtained from the timelike region, in both the hard-wall and soft-wall models. Our results showed that both models predict $Q^{2}$ dependence for $F_{\pi}\left(Q^{2}\right)$ that tends to be too shallow for both models (in particular, predicting a value too small for the charge radius $\left\langle r_{\pi}^{2}\right\rangle$ ), but significantly worse for the soft-wall case. Better agreement with $F_{\pi}\left(Q^{2}\right)$ data could be achieved only by loosening the fit to other observables, especially by decreasing the decay constant $f_{\pi}$.

However, a small swindle is introduced in applying the methods of Ref. [5] to the softwall model [4]: The background scalar field $X(z)$ whose vacuum expectation value $X_{0}(z)$ provides $m_{q}$ and $\sigma$ via its small- $z$ asymptotic value $\left(2 X_{0}(z)=m_{q} z+\sigma z^{3}\right)$ satisfies in general a 2nd-order differential equation whose solutions are Kummer functions, only one of which $\left[z U\left(\frac{1}{2}, 0, \kappa^{2} z^{2}\right)\right.$, to be precise $]$ satisfies the necessary finiteness boundary condition of the soft-wall model as $z \rightarrow \infty$. But this particular function carries a unique proportionality between the $z$ and $z^{3}$ terms of its Taylor series, implying an unphysical fixed ratio between $m_{q}$ and $\sigma$. Such an obstacle is easily overcome by including higher-order terms in $X(z)$ in the potential that do not greatly modify the small- $z$ behavior; however, from the operational point of view, in Ref. [24] we simply observed that such large- $z$ modifications are numerically heavily suppressed by the background factor $e^{-\kappa^{2} z^{2}}$. One of the goals of the current work is to present a calculation using a field $X_{0}(z)$ that satisfies both constraints, finite for large $z$ and allowing independent $m_{q}$ and $\sigma$ values. Numerous functional forms for the field $X_{0}(z)$ satisfy this requirement, but for sake of definiteness we choose

$$
2 X_{0}(z)=\left(m_{q} z+\sigma z^{3}\right)\left[1-\exp \left(-\frac{A_{c}}{\kappa^{4} z^{4}}\right)\right]+B_{c} \exp \left(-\frac{3}{4 \kappa^{2} z^{2}}\right) .
$$

We discuss the motivation for this particular choice in Sec. III, but point out that numerical simulations for several other similar forms do not significantly alter our conclusion: Using a functional form satisfying all required asymptotic behaviors does not significantly change the naive soft-wall fit of Ref. [24].

The other goal of this paper is to develop a model that combines the numerical successes of the hard-wall model with the Regge trajectories predicted by the soft-wall model. In the closing statements of Ref. [24] we presented a background field with precisely these properties. Inspired by the old Saxon-Woods generalization of the hard-cutoff nuclear density 
model, we proposed

$$
e^{-\Phi(z)}=\frac{e^{\lambda^{2} z_{0}^{2}}-1}{e^{\lambda^{2} z_{0}^{2}}+e^{\lambda^{2} z^{2}}-2},
$$

which has a drop-off at $z=z_{0}$ (and equals $\frac{1}{2}$ there), but decreases as $e^{-\lambda^{2} z^{2}}$ for large $z$.

Our results indicate that neither of these modifications substantially alter the outcome of Ref. [24]. The soft-wall model test function $X_{0}(z)$ of Eq. (2), although formally possessing the correct asymptotic behaviors, gives an optimal fit to $F_{\pi}\left(Q^{2}\right)$ no better than that in Ref. [24]. Similarly, as expected, the adoption of Eq. (3) for the background field interpolates results between hard- and soft-wall models. This latter result may be somewhat surprising since Eq. (3) contains two independent mass parameters $\left(1 / z_{0}\right.$ and $\left.\lambda\right)$ rather than the one of the strict hard- or soft-wall models. Since our previous work [24] showed that both hard- and soft-wall model predictions for $F_{\pi}\left(Q^{2}\right)$ can be improved significantly by loosening the fit to just one observable, one might expect that Eq. (3) with its extra fit parameter allows sufficient latitude not only to give both hard- and soft-wall behaviors, but also to improve the global numerical fit to $F_{\pi}\left(Q^{2}\right)$ and other low-energy observables. As seen below, the former goal is achieved but the latter is not. Taking these results together, it appears that achieving a better simultaneous fit to $F_{\pi}\left(Q^{2}\right), f_{\pi}, m_{\pi}, m_{\rho}, f_{\rho}$, and other low-energy observables requires a more general treatment of chiral symmetry breaking than the one developed in Ref. [5].

This paper is organized as follows: In Sec. II we recount the formalism of Ref. [5] relevant to our calculations and give expressions for $F_{\pi}\left(Q^{2}\right)$ and couplings in terms of AdS/QCD mode wave functions. Section III presents the results of numerical simulations of $F_{\pi}\left(Q^{2}\right)$ and other low-energy hadronic observables, and Sec. IV summarizes our results and concludes. Details of the numerical procedure are discussed in the Appendix.

\section{FORMALISM}

The content of this section is nearly identical to that in Ref. [24], but is included for completeness. The full 5D action [5] used in this work reads

$$
S=\int d^{5} x e^{-\Phi(z)} \sqrt{g} \operatorname{Tr}\left\{|D X|^{2}+3|X|^{2}-\frac{1}{4 g_{5}^{2}}\left(F_{L}^{2}+F_{R}^{2}\right)\right\},
$$

where $g \equiv\left|\operatorname{det} g_{M N}\right|$ is obtained from the metric in Eq. (11), and $e^{-\Phi(z)}$ represents a background dilaton coupling. The original holographic QCD calculation in Ref. [5] uses the 
hard-wall (step function) background $e^{-\Phi(z)}=H\left(z_{0}-z\right)$, while Ref. [4] defines the soft-wall model by $e^{-\Phi(z)}=e^{-\kappa^{2} z^{2}}$, which as noted above, reproduces the traditional Regge trajectory behavior for the mesons. In this paper we also consider the Saxon-Woods form for $e^{-\Phi(z)}$ defined in Eq. (3). The scalar field $X(z)$ [actually $(2 / z) X(z)$ ] is the holographic partner of the quark condensate $\bar{q}_{R} q_{L}$, and its vacuum expectation value $X_{0}(z) \equiv \frac{1}{2} v(z)=\frac{1}{2} m_{q} z+\frac{1}{2} \sigma z^{3}$ gives rise to explicit and spontaneous chiral symmetry breaking. This expression for $X_{0}(z)$ is exact in the hard-wall model, while it holds only for small $z$ in the soft-wall model.

The chiral gauge fields $A_{L, R}^{a}$ (holographic partners to the bilinears $\bar{q}_{L, R} \gamma^{\mu} t^{a} q_{L, R}$ ) enter through $D^{M} X \equiv \partial^{M} X-i A_{L}^{M} X+i X A_{R}^{M}, A_{L, R}^{M} \equiv A_{L, R}^{M a} t^{a}$, and $F_{L, R}^{M N} \equiv \partial^{M} A_{L, R}^{N}-\partial^{N} A_{L, R}^{M}-$ $i\left[A_{L, R}^{M}, A_{L, R}^{N}\right]$. The polar $V$ and axial $A$ gauge fields are $V^{M} \equiv \frac{1}{2}\left(A_{L}^{M}+A_{R}^{M}\right)$ and $A^{M} \equiv$ $\frac{1}{2}\left(A_{L}^{M}-A_{R}^{M}\right)$, in terms of which $D^{M} X=\partial^{M} X-i\left[V^{M}, X\right]-i\left\{A^{M}, X\right\}, F_{V}^{M N} \equiv \partial^{M} V^{N}-\partial^{N} V^{M}-$ $i\left(\left[V^{M}, V^{N}\right]+\left[A^{M}, A^{N}\right]\right), F_{A}^{M N} \equiv \partial^{M} A^{N}-\partial^{N} A^{M}-i\left(\left[V^{M}, A^{N}\right]+\left[A^{M}, V^{N}\right]\right)$, and

$$
S=\int d^{5} x e^{-\Phi(z)} \sqrt{g} \operatorname{Tr}\left\{|D X|^{2}+3|X|^{2}-\frac{1}{2 g_{5}^{2}}\left(F_{V}^{2}+F_{A}^{2}\right)\right\} .
$$

We use the axial-like gauge $V_{z}(x, z)=0, A_{z}(x, z)=0$, so that the associated sources may be expressed as divergences over just the usual four spacetime dimensions, $\partial^{\mu} V_{\mu}=0$ (since isospin is conserved) and $\partial^{\mu} A_{\mu}$. $A_{\mu}$ can be further decomposed into a transverse (divergenceless) piece $A_{\mu \perp}$ and a longitudinal piece $\varphi: A_{\mu}=A_{\mu \perp}+\partial_{\mu} \varphi$. The pion field $\pi^{a}$ appears through $X=X_{0} \exp \left(2 i \pi^{a} t^{a}\right) ; \pi^{a}$ is dimensionless and related to the canonically-normalized pion field $\tilde{\pi}^{a}$ of chiral Lagrangians via $\pi^{a}=\tilde{\pi}^{a} / f_{\pi}$, with $f_{\pi}=93 \mathrm{MeV}$.

The equations of motion obtained from Eq. (5) for the fields $\Psi(q, z)$ (i.e., all except for $X_{0}$ are Fourier transformed with respect to the $4 \mathrm{D}$ coordinates $x$ ) read

$$
\begin{gathered}
\partial_{z}\left(\frac{e^{-\Phi(z)}}{z} \partial_{z} V_{\mu}^{a}\right)+\frac{q^{2} e^{-\Phi(z)}}{z} V_{\mu}^{a}=0 \\
{\left[\partial_{z}\left(\frac{e^{-\Phi(z)}}{z} \partial_{z} A_{\mu}^{a}\right)+\frac{q^{2} e^{-\Phi(z)}}{z} A_{\mu}^{a}-\frac{g_{5}^{2} v(z)^{2} e^{-\Phi(z)}}{z^{3}} A_{\mu}^{a}\right]_{\perp}=0} \\
\partial_{z}\left(\frac{e^{-\Phi(z)}}{z} \partial_{z} \varphi^{a}\right)+\frac{g_{5}^{2} v(z)^{2} e^{-\Phi(z)}}{z^{3}}\left(\pi^{a}-\varphi^{a}\right)=0 \\
-q^{2} \partial_{z} \varphi^{a}+\frac{g_{5}^{2} v(z)^{2}}{z^{2}} \partial_{z} \pi^{a}=0 \\
\partial_{z}\left(\frac{e^{-\Phi(z)}}{z^{3}} \partial_{z} X_{0}\right)+\frac{3 e^{-\Phi(z)}}{z^{5}} X_{0}=0 .
\end{gathered}
$$


The gauge/gravity correspondence itself provides the first prediction, the value of the 5D gauge coupling $g_{5}[5]$. Substituting Eq. (6) into the $F_{V}^{2}$ portion of Eq. (5) leaves only the boundary term for the action:

$$
S=-\left.\frac{1}{2 g_{5}^{2}} \int d^{4} x \frac{e^{-\Phi(z)}}{z} V_{\mu}^{a} \partial_{z} V^{\mu a}\right|_{z=\epsilon} .
$$

The vector field $V_{\mu}^{a}(q, z)$ can be resolved as $V_{\mu}^{a}(q, z)=V(q, z) \tilde{V}_{\mu}^{a}(q)$, where $\tilde{V}_{\mu}^{a}(q)$ is the Fourier transform of the vector current source $J_{\mu}^{a}=\bar{q} \gamma_{\mu} t^{a} q$ at the UV boundary $z=\epsilon$, and $V(q, z)$ (the "bulk-to-boundary propagator") is normalized to $V(q, \epsilon)=1$. Due to the isospin conservation constraint $q_{\mu} V^{\mu}=0$, one may replace $\tilde{V}_{\mu}^{a} \tilde{V}^{\mu a}$ with $\tilde{V}_{\mu}^{a} \tilde{V}_{\nu}^{b} \Pi^{\mu \nu} \delta^{a b}$ and $\Pi^{\mu \nu} \equiv \eta^{\mu \nu}-q^{\mu} q^{\nu} / q^{2}$, and then the usual quadratic variation of the action with respect to the source $\tilde{V}$ produces the vector current two-point function:

$$
\begin{aligned}
\int d^{4} x e^{i q x}\left\langle J_{\mu}^{a}(x) J_{\nu}^{b}(0)\right\rangle & =\delta^{a b} \Pi_{\mu \nu} \Sigma_{V}\left(q^{2}\right), \\
\Sigma_{V}\left(q^{2}\right) & =-\left.\frac{e^{-\Phi(z)}}{g_{5}^{2}} \frac{\partial_{z} V(q, z)}{z}\right|_{z=\epsilon},
\end{aligned}
$$

from which one finds, matching to the QCD result for currents $J_{\mu}$ normalized [19] according to the prescription of [5],

$$
g_{5}^{2}=\frac{12 \pi^{2}}{N_{c}} \rightarrow 4 \pi^{2}
$$

An analogous calculation in the axial sector relates the bulk-to-boundary propagator $A(q, z)$ to the $\pi$ decay constant $f_{\pi}$ :

$$
f_{\pi}^{2}=-\left.\frac{1}{g_{5}^{2}} \frac{\partial_{z} A(0, z)}{z}\right|_{z=\epsilon}
$$

from which one sees that the relative choices of normalization for $f_{\pi}$ and $g_{5}$ are correlated.

The normalizable eigenstates of Eqs. (6) -(9) correspond to towers of hadrons of the same quantum numbers as the parent fields. Since large $N_{c}$ is intrinsic to this procedure, the mesons have narrow widths and the spectral decompositions of self-energy functions such as $\Sigma_{V}$ are sums over poles:

$$
\Sigma_{V}\left(q^{2}\right)=\sum_{n=0}^{\infty} \frac{f_{n}^{2}}{q^{2}-M_{n}^{2}},
$$

where $M_{n}$ are the mass eigenvalues and $f_{n}$ are the decay constants of vector modes $\psi_{n}(z)$ normalized according to

$$
\int d z \frac{e^{-\Phi(z)}}{z} \psi_{m}(z) \psi_{n}(z)=\delta_{m n} .
$$


In order to obtain the pion form factor $F_{\pi}$, one expands the action Eq. (5) out to cubic order in the fields. Since the pion field is related [Eq. (91)] to the longitudinal mode $\partial^{\mu} \varphi$ of $A^{\mu}$, one must identify not only $V \pi \pi$ terms, but also $V A A$ and $V A \pi$. A straightforward calculation (that uses the equation of motion for $V$ to simplify the result) produces the $V \pi \pi$ terms:

$$
\begin{aligned}
S_{\mathrm{AdS}}^{V \pi \pi}= & \epsilon_{a b c} \int d^{4} x \int d z e^{-\Phi(z)}\left[\frac{1}{g_{5}^{2} z}\left(\partial_{z} \partial^{\mu} \varphi^{a}\right) V_{\mu}^{b}\left(\partial_{z} \varphi^{c}\right)\right. \\
& \left.+\frac{v(z)^{2}}{z^{3}}\left(\partial^{\mu} \pi^{a}-\partial^{\mu} \varphi^{a}\right) V_{\mu}^{b}\left(\pi^{c}-\varphi^{c}\right)\right],
\end{aligned}
$$

where the $z$ integration range is $[0, \infty)$. Reference [5] uses this action (but only with $e^{-\Phi(z)}=$ 1) to obtain the $V \pi \pi$ couplings [Eq. (25) below], with the caveat that terms cubic in $F_{V, A}$ have not been included. In fact, we have shown [24] that no such terms contribute to the $V \pi \pi$ coupling. The 3-point correlator is obtained from a straightforward variation of Eq. (18):

$$
\left\langle J_{\pi}^{a}\left(p_{1}\right) J_{V}^{\mu, b}(q) J_{\pi}^{c}\left(-p_{2}\right)\right\rangle=\epsilon^{a b c} F\left(p_{1}^{2}, p_{2}^{2}, q^{2}\right)\left(p_{1}+p_{2}\right)^{\mu} i(2 \pi)^{4} \delta^{(4)}\left(p_{1}-p_{2}+q\right),
$$

where, again recalling the narrowness of resonances, one may express the dynamical factor $F\left(p_{1}^{2}, p_{2}^{2}, q^{2}\right)$ in terms of transition form factors:

$$
F\left(p_{1}^{2}, p_{2}^{2}, q^{2}\right)=\sum_{n, k=1}^{\infty} \frac{f_{n} f_{k} F_{n k}\left(q^{2}\right)}{\left(p_{1}^{2}-M_{n}^{2}\right)\left(p_{2}^{2}-M_{k}^{2}\right)}
$$

and $F_{n k}\left(q^{2}\right)$ correspond to form factors for $n \rightarrow k$ transitions. The pion form factor $F_{\pi}\left(q^{2}\right)$ is then defined as the ground-to-ground pseudoscalar meson transition to the vector current:

$$
F_{\pi}\left(q^{2}\right) \equiv F_{11}\left(q^{2}\right)=\int d z e^{-\Phi(z)} \frac{V(q, z)}{f_{\pi}^{2}}\left\{\frac{1}{g_{5}^{2} z}\left[\partial_{z} \varphi(z)\right]^{2}+\frac{v(z)^{2}}{z^{3}}[\pi(z)-\varphi(z)]^{2}\right\}
$$

whose normalization $F_{\pi}(0)=1[\operatorname{using} V(0, z)=1]$ is guaranteed by the canonical normalization of the pion kinetic energy term in the 4D Lagrangian. The origin of the $z$ dependence of Eq. (21) is recognizable from Eq. (18). The pion is the ground-state solution of Eqs. (8) -(9) for $\pi^{a}$ subject to appropriate boundary constraints: Neumann at $z=z_{0}$ for the hard-wall model, finite as $z \rightarrow \infty$ for the soft-wall model. The solution to Eq. (6) for source $V$ in the soft-wall model $\left[\Phi(z)=\kappa^{2} z^{2}\right]$ for spacelike momentum transfers $q^{2} \equiv-Q^{2}<0$ [19, 22, 24], used in our first calculation, includes the Kummer (confluent hypergeometric) function $U$ :

$$
V(q, z)=\Gamma\left(1+Q^{2} / 4 \kappa^{2}\right) U\left[Q^{2} / 4 \kappa^{2}, 0,(\kappa z)^{2}\right]
$$


Note that for large $z$, Eq. (22) falls as $\left(z^{2}\right)^{-Q^{2} / 4 \kappa^{2}}$. For our second calculation $\left[e^{-\Phi(z)}\right.$ as in Eq. (3) ], $V(q, z)$ must be obtained numerically from Eq. (6). In both cases the solutions satisfy the boundary conditions $V(q, \epsilon)=1, V(0, z)=1$. In the timelike region, $V(q, z)$ may be expanded as

$$
V(q, z)=-g_{5} \sum_{n=1}^{\infty} \frac{f_{n} \psi_{n}(z)}{q^{2}-M_{n}^{2}} .
$$

When numerical solutions are required, $\psi_{n}(z)$ and $f_{n}$ are obtained by solving Eq. (6) at its

poles and properly normalizing using Eq. (17). In either case, substituting Eq. (23) into Eq. (21) gives the timelike pion form factor as a sum over vector meson poles:

$$
F_{\pi}\left(q^{2}\right)=-\sum_{n=1}^{\infty} \frac{f_{n} g_{n \pi \pi}}{q^{2}-M_{n}^{2}}
$$

where $g_{n \pi \pi}$ is given by

$$
g_{n \pi \pi}=\frac{g_{5}}{f_{\pi}^{2}} \int d z \psi_{n}(z) e^{-\Phi(z)}\left\{\frac{1}{g_{5}^{2} z}\left[\partial_{z} \varphi(z)\right]^{2}+\frac{v(z)^{2}}{z^{3}}[\pi(z)-\varphi(z)]^{2}\right\} .
$$

Together, Eqs. (21) and Eqs. (24) -(25), with $V$ and $\psi_{n}$ obtained from either Eq. (22) or the numerical solution to Eq. ([6) $)$, provide a complete expression for $F_{\pi}\left(q^{2}\right)$ at all values of $q^{2}$.

\section{RESULTS}

For our first result, we consider the consequences of modifying the solution for the vev of the bulk field $X(z)$, as stated in the Introduction and repeated here for convenience:

$$
2 X_{0}(z) \equiv v(z)=\left(m_{q} z+\sigma z^{3}\right)\left[1-\exp \left(-\frac{A_{c}}{\kappa^{4} z^{4}}\right)\right]+B_{c} \exp \left(-\frac{3}{4 \kappa^{2} z^{2}}\right)
$$

This particular expression is chosen because of the interesting large- $z$ asymptotic form of solutions of Eq. (10) for $X_{0}(z)$ [4]: In the hard-wall case the independent solutions are precisely $z^{1}$ and $z^{3}$ (so that the asymptotic solution is also the full solution in that case), while for the soft-wall case the exact solutions are the Kummer functions $z^{3} M\left(\frac{3}{2}, 2, \kappa^{2} z^{2}\right)$ and $z U\left(\frac{1}{2}, 0, \kappa^{2} z^{2}\right)$. Of the latter pair only the second is finite as $z \rightarrow \infty$, and indeed behaves as $\exp \left(-3 / 4 \kappa^{2} z^{2}\right)$. As indicated in the Introduction, $X_{0}$ must have corrections arising from higher-order terms in the potential to allow independent $m_{q}$ and $\sigma$ parameters at low $z$, which in turn would appear as corrections to Eq. (10). This argument originated with the original soft-wall paper [4], and since a full explanation that motivates our choice Eq. (2) requires 
understanding the original argument in detail, we repeat it here. Inasmuch as $e^{-\Phi(z)}$ vanishes as $z \rightarrow \infty$ in the soft-wall model, the equation of motion Eq. (10) for $X_{0}$ is guaranteed to possess a solution that tends to a constant as $z \rightarrow \infty$. Since the $e^{-\Phi(z)}$ factor also multiplies any higher-order potential terms in $X$ that might be introduced into the action Eq. (5) and hence into Eq. (10), the full equation of motion continues to possess a solution that approaches a constant as $z \rightarrow \infty$. Moreover, for such a solution with $X_{0}(\infty)=$ constant, all multiple-derivative terms appearing in Eq. (10) and its generalizations become negligible compared to the lone single-derivative term contained in the first term of Eq. (10). The constant $X_{0}(\infty)$ therefore acts as the sole integration constant in this effectively 1st-order differential equation, and thus determines the (in general nonlinear) relationship between its solution's $z^{1}$ and $z^{3}$ coefficients, namely, the ratio of $m_{q}$ to $\sigma$. By adjusting the higher-order terms in the potential, one changes $X_{0}(\infty)$, thus altering the numerical ratio between its coefficients near $z=0$, thereby allowing one to obtain the desired ratio of $m_{q}$ to $\sigma$.

With these features in mind, let us return to the motivation for the choice Eq. (2). The asymptotic form $\exp \left(-3 / 4 \kappa^{2} z^{2}\right)$ already appears for the explicit solution to Eq. (10) and therefore is chosen for the $z \rightarrow \infty$ limit of Eq. (2). The functional forms $\exp \left(-A / z^{n}\right)$ with $A, n>0$ are especially interesting because they not only rapidly approach 1 as $z \rightarrow \infty$, but also sluggishly depart from 0 as $z$ is increased from 0 , due to their nonanalyticity about that point. Thus the small- $z$ asymptotic form of Eq. (2), $m_{q} z+\sigma z^{3}$, is precisely as required. These properties make Eq. (2) an excellent choice for a test function satisfying the desired asymptotic behaviors. Note that we do not exhibit a specific potential with this solution to its equation of motion, since Eq. (2) satisfies all the requirements placed upon it through its solution. Moreover, Eq. (2) gives results for $F_{\pi}\left(Q^{2}\right)$ and other observables in particular ranges of its parameters $A_{c}$ and $B_{c}$ that agree numerically with the original soft-wall model (which, recall, is the exact solution for a particular potential), thereby providing additional (a posteriori) support for this choice. In particular, the precise form of $X_{0}$ at intermediate values of $z$-ignored in the construction of Eq. (2) - is apparently not important in fitting observables.

As mentioned above, the numerical change from the results of Ref. [24] (the original softwall model curve in Fig. 1) due to using Eq. (2) turns out to be quite minimal in the best-fit case. For sake of definiteness, let us choose $B_{c}=1$ and $A_{c}=1$. We find these values give a best fit to $F_{\pi}\left(Q^{2}\right)$ data quite close to (but slightly worse than) that of the original soft-wall 
TABLE I: Comparison of soft-wall model to modified $e^{-\Phi(z)}$ with $\lambda z_{0}=1$; values in MeV (except for $\left.g_{\rho \pi \pi}\right)$.

\begin{tabular}{cccc}
\hline \hline Observable & Experiment & Soft-wall & $\lambda z_{0}=1$ \\
\hline$m_{\pi}$ & $139.6 \pm 0.0004[25]$ & 139.6 & 139.6 \\
$m_{\rho}$ & $775.5 \pm 0.4[25]$ & 777.4 & 779.2 \\
$m_{a_{1}}$ & $1230 \pm 40[25]$ & 1601 & 1596 \\
$f_{\pi}$ & $92.4 \pm 0.35[25]$ & 87.0 & 92.0 \\
$f_{\rho}^{1 / 2}$ & $346.2 \pm 1.4[26]$ & 261 & 283 \\
$f_{a_{1}}^{1 / 2}$ & $433 \pm 13[27,28]$ & 558 & 576 \\
$g_{\rho \pi \pi}$ & $6.03 \pm 0.07[25]$ & 3.33 & 3.49 \\
\hline \hline
\end{tabular}

model obtained in Ref. [24] - an encouraging start. Keeping $A_{c}=1$ and varying $B_{c}$, we find that values of $B_{c}<1$ (including $B_{c}<0$ ) give an $F_{\pi}$ prediction that moves even further away from the data (i.e., is even more shallow) than the original soft-wall prediction. On the other hand, for $B_{c}>1$ the prediction moves closer to $F_{\pi}$ data, but still is only as good as the original soft-wall prediction at $B_{c} \approx 2$. For $B_{c} \gtrsim 2$ the prediction again moves away from the $F_{\pi}$ data. Next, if we keep $B_{c}=1$ and vary $A_{c}$, we find that for $0<A_{c}<1$ the prediction moves away from the $F_{\pi}$ data, but for $A_{c}>1$ the prediction improves only marginally. Interestingly, letting both $B_{c}$ and $A_{c}$ vary always appears to permit a set of parameters that fit the data $\left[F_{\pi}\right.$ and the static observables $m_{\pi}, m_{\rho}, m_{a_{1}}, f_{\pi}, f_{\rho}^{1 / 2}, f_{a_{1}}^{1 / 2}$, and $\left.g_{\rho \pi \pi}\right]$ reasonably well, but only if $B_{c} \lesssim 2$. However, these fits are never superior to the original soft-wall fit. We conclude that the original fit in Ref. [24], although formally violating the AdS/QCD boundary conditions, is actually quite stable when the proper boundary conditions are imposed.

That the new fits never improve upon the original model is quite easy to explain numerically. First note that $V(q, z)$ in Eq. (22) drops from 1 at $z=0$ to smaller values for larger $z$ with increasing rapidity as $Q^{2}$ increases, a feature not affected by our modification to $X_{0}(z)$. The effect of the new $X_{0}(z)$ is reflected only in the rest of integrand in Eq. (21), whose integral [i.e., for $V(q, z) \rightarrow 1$ ] is normalized to 1 . One expects that the integrand favors larger $z$ in the original soft-wall case because of the direct $z^{3}$ dependence of $X_{0}(z)$ than in the modified case, since $X_{0}(z) \rightarrow B_{c}$ as $z \rightarrow \infty$. Since $V(q, z)$ decreases with $z$ for all $Q^{2}>0, F_{\pi}\left(Q^{2}\right)$ is also lower in the original model for all values of $Q^{2}>0$. Our numerical 
TABLE II: Comparison of hard-wall model to the $e^{-\Phi(z)}$ of Eq. (3) with $\lambda z_{0}=2.1$; values in $\mathrm{MeV}$ (except for $\left.g_{\rho \pi \pi}\right)$.

\begin{tabular}{cccc}
\hline \hline Observable & Experiment & Hard wall & $\lambda z_{0}=2.1$ \\
\hline$m_{\pi}$ & $139.6 \pm 0.0004[25]$ & 139.6 & 139.6 \\
$m_{\rho}$ & $775.5 \pm 0.4[25]$ & 775.3 & 777.5 \\
$m_{a_{1}}$ & $1230 \pm 40[25]$ & 1358 & 1343 \\
$f_{\pi}$ & $92.4 \pm 0.35[25]$ & 92.1 & 88.0 \\
$f_{\rho}^{1 / 2}$ & $346.2 \pm 1.4[26]$ & 329 & 325 \\
$f_{a_{1}}^{1 / 2}$ & $433 \pm 13[27,28]$ & 463 & 474 \\
$g_{\rho \pi \pi}$ & $6.03 \pm 0.07[25]$ & 4.48 & 4.63 \\
\hline \hline
\end{tabular}

simulation indeed verifies this behavior. The $F_{\pi}\left(Q^{2}\right)$ prediction using the modified $X_{0}(z)$ in Eq. (2) is never smaller than that of the original soft-wall model, and hence lies at least as far from the $F_{\pi}\left(Q^{2}\right)$ data.

Second, we explore the consequences of modifying the background field as in Eq. (3), repeated here for convenience:

$$
e^{-\Phi(z)}=\frac{e^{\lambda^{2} z_{0}^{2}}-1}{e^{\lambda^{2} z_{0}^{2}}+e^{\lambda^{2} z^{2}}-2} .
$$

As expected, the parameter $\lambda$ serves to interpolate between the hard-wall and soft-wall models. Specifically, for $\lambda z_{0} \approx 1$ we find that the model prediction for $F_{\pi}$ is very similar to the original soft-wall result, while for $\lambda z_{0} \approx 2$ the prediction is very close to the original hardwall result: See Fig. 1. Beyond $\lambda z_{0} \approx 2$ the global fit does not improve much; in particular, the $F_{\pi}\left(Q^{2}\right)$ fit is best for $\lambda z_{0}=2.1$, but at the cost of $f_{\pi}=88.0 \mathrm{MeV}$, while at $\lambda z_{0}=2.4$ the $F_{\pi}\left(Q^{2}\right)$ fit is not very good but one obtains $f_{\pi}=92.7 \mathrm{MeV}$. That results mimicking the older models are obtained for such modest values of $\lambda z_{0}$ is especially interesting because the hard-wall limit is formally recovered only when $\lambda z_{0} \rightarrow \infty$, and the soft-wall limit is formally recovered only when $\lambda z \gg \lambda z_{0}, 1$.

As a comparison, we present results for both $\lambda z_{0}=1$ and $\lambda z_{0}=2.1$ as well as the original soft- and hard-wall predictions in Tables II and II, respectively. Even though the result for $\lambda z_{0} \approx 2$ is very similar to original hard-wall result, its predictions for the positions of higher-order vector meson poles differ strongly from those of the original hard-wall model 


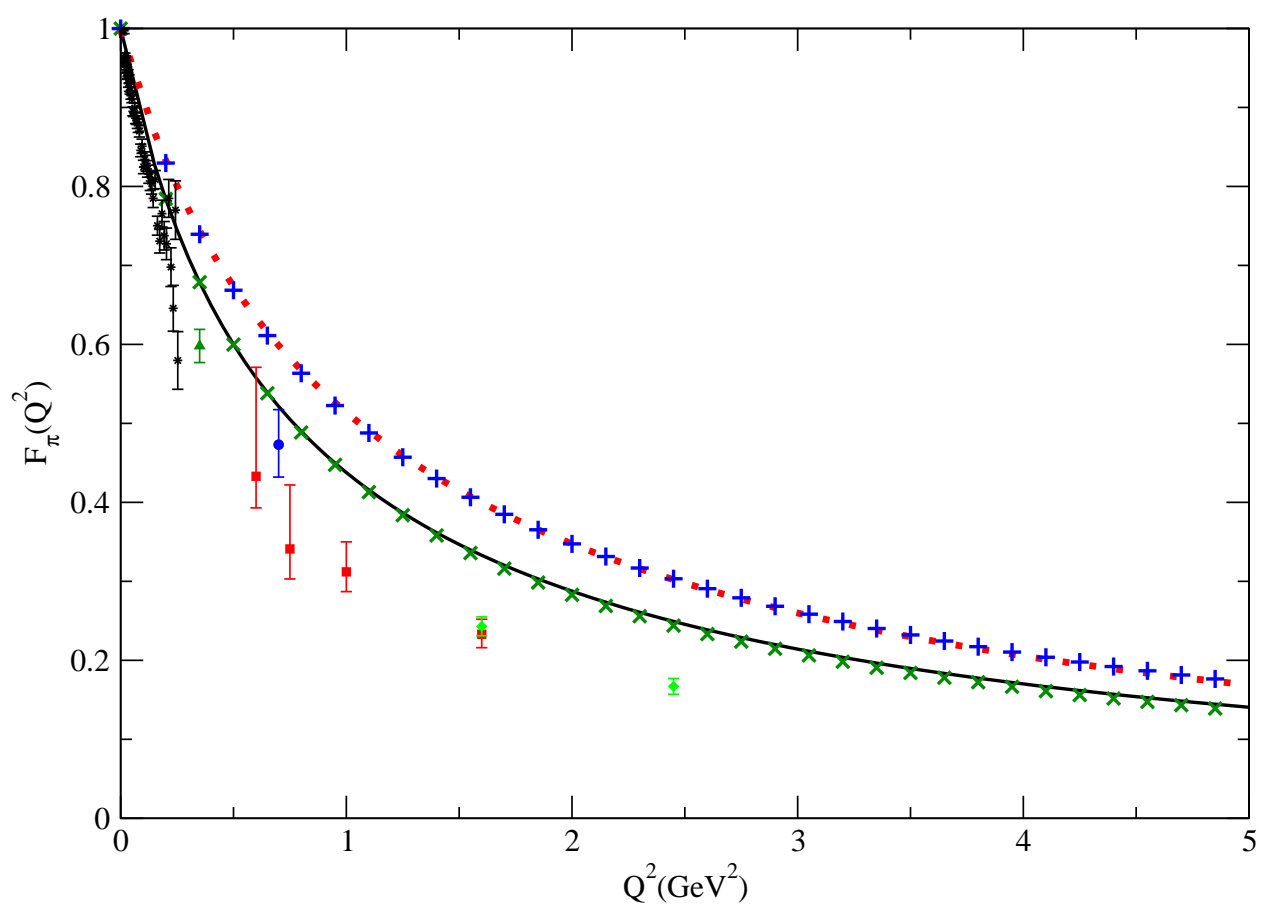

FIG. 1: Spacelike scaling behavior for $F_{\pi}\left(Q^{2}\right)$ as a function of $Q^{2}=-q^{2}$. The continuous line is the prediction of the original hard-wall model with $1 / z_{0}=323 \mathrm{MeV}$. The dotted line is the prediction of the original soft-wall model with $\kappa=m_{\rho} / 2$. The crosses use $e^{-\Phi(z)}$ of Eq. (3) with $\lambda z_{0}=2.1$, and the pluses use $\lambda z_{0}=1$. The stars are from a data compilation from CERN [29], the circles are from DESY, reanalyzed by Tadevosyan et al. [30, 31], the triangle is data from DESY [32], and the boxes [31] and diamonds [33] are from Jefferson Lab. Older data in the range 3-10 $\mathrm{GeV}^{2}$ [34] exist but have large uncertainties and are not presented here.

(Table 【II). The modified $e^{-\Phi(z)}$ results exhibit a closer match to a $m_{n}^{2} \sim n$ vector meson Regge trajectory, in contrast to the $m_{n}^{2} \sim n^{2}$ scaling of the original hard-wall trajectory. From Table III one finds in the hard-wall case that $m_{n+1}-m_{n}$ is stable around $1010 \mathrm{MeV}$, while in the modified background case, $\left(m_{n+1}^{2}-m_{n}^{2}\right)^{1 / 2}$ is stable around $1450 \mathrm{MeV}$. We conclude that the $e^{-\lambda^{2} z^{2}}$ tail behavior of Eq. (3) is already significant when $\lambda z_{0} \approx 2$, where the prediction for $F_{\pi}\left(Q^{2}\right)$ closely matches the hard-wall result. We have thus produced a model that works as well as the hard-wall model in fitting to the low-energy data, but that in addition reproduces the appropriate Regge trajectories expected from linear confinement.

For completeness we also include the pion charge radius $\left\langle r_{\pi}^{2}\right\rangle$ results for both models. In the modified $X_{0}$ model for $B_{c}=0.96$ and $A_{c}=1.1$, we find $\left\langle r_{\pi}^{2}\right\rangle=(0.485 \mathrm{fm})^{2}$. In the 
TABLE III: Comparison of vector meson masses and decay constants (in MeV) for the hard-wall model and the model using $e^{-\Phi(z)}$ of Eq. (3) with $\lambda z_{0}=2.1$.

\begin{tabular}{cccc}
\hline \hline \multicolumn{2}{c}{ Original hard wall } & \multicolumn{2}{c}{$e^{-\Phi(z)}$ from Eq. (3) with $\lambda z_{0}=2.1$} \\
\hline$m_{\rho}$ & $F_{\rho}^{1 / 2}$ & $m_{\rho}$ & $F_{\rho}^{1 / 2}$ \\
\hline 775.6 & 329 & 777.5 & 325 \\
1780.2 & 616 & 1608.1 & 528 \\
2790.8 & 864 & 2226.8 & 611 \\
3802.8 & 1089 & 2637.5 & 644 \\
4815.2 & 1300 & 2986.6 & 683 \\
\hline \hline
\end{tabular}

modified $e^{-\Phi}$ model for $\lambda z_{0}=1.0,\left\langle r_{\pi}^{2}\right\rangle=(0.500 \mathrm{fm})^{2}$, while for $\lambda z_{0}=2.1$ it is $(0.576 \mathrm{fm})^{2}$. In comparison, the original soft-wall model gives $\left\langle r_{\pi}^{2}\right\rangle=(0.494 \mathrm{fm})^{2}$, the original hard-wall model gives $\left\langle r_{\pi}^{2}\right\rangle=(0.576 \mathrm{fm})^{2}$, and the experimental value is $\left\langle r_{\pi}^{2}\right\rangle=[0.672(8) \mathrm{fm}]^{2}[25]$.

Having addressed the two central issues of this paper, we now discuss an even more interesting conundrum, namely, the fact that all the fits described here are limited in how closely they approach the full set of $F_{\pi}\left(Q^{2}\right)$ and other low-energy data. All of them produce curves too shallow in $Q^{2}$, leading in particular to a value of $\left\langle r_{\pi}^{2}\right\rangle$ smaller than experiment. We observed in Ref. [24] that a better fit to $F_{\pi}\left(Q^{2}\right)$ could be obtained by loosening the fit to the other low-energy observables, particularly by letting $f_{\pi}$ be somewhat smaller, a resolution that is not entirely satisfactory. However, as is well known [35], the functional behavior of $F_{\pi}\left(Q^{2}\right)$ for large $Q^{2}$ is determined by its partonic substructure: $F_{\pi}\left(Q^{2}\right) \propto f_{\pi}^{2} \alpha_{s}\left(Q^{2}\right) / Q^{2}$. The AdS/QCD model used here contains only hadronic and no explicit partonic degrees of freedom, so it is perhaps not surprising that the $F_{\pi}\left(Q^{2}\right)$ prediction has difficulty falling as steeply as the data for larger $Q^{2}$. But are partons essential at the relatively small values $\left(Q^{2} \leq 5 \mathrm{GeV}^{2}\right)$ presented in Fig. [1] If not, then we are faced with the possibility that the pattern of chiral symmetry breaking developed in Ref. [5] and represented by the background field $X_{0}(z)$ may require improvement. 


\section{CONCLUSIONS}

In this paper we have shown, first, that the treatment in Ref. [24] of chiral symmetry breaking in the soft-wall model, although not formally satisfying all the boundary conditions of that model, nevertheless gives numerical results in agreement with those of models possessing all the correct asymptotic behaviors. Indeed, we find that the soft-wall calculation of Ref. [24] appears to represent a best-fit limit to such improved models.

We have also developed a model that successfully interpolates between hard-wall and soft-wall models: It reproduces the desirable numerical features of the hard-wall model but also the Regge behavior predicted by linear confinement that motivates the soft-wall model. The fit to static observables as well as $F_{\pi}\left(Q^{2}\right)$ is as good as that from the hard-wall model, but the excited $\rho$ masses now follow the desired pattern $m_{n}^{2} \sim n$.

Nevertheless, all of the models proposed here and in Ref. [24] share the properties that their predictions for $F_{\pi}\left(Q^{2}\right)$ are too shallow for larger $Q^{2}$ values, unless one relaxes the fit to one or more of the static observables (particularly $f_{\pi}$ ). We have argued that the most likely culprits for this discrepancy are either the application of a purely hadronic AdS/QCD model to a region (large $Q^{2}$ ) where partonic degrees of freedom become appreciable, or the need for an improvement to the pattern of chiral symmetry breaking introduced via background fields. Both directions are well worthy of further research.

\section{APPENDIX: NUMERICAL PROCEDURE}

Solutions to Eqs. (6) -(10) are obtained numerically using subroutines provided by $\mathrm{Nu}$ merical Recipes (NR) [36], and all terminology provided in this appendix is discussed in that well-known standard reference. These differential equations are solved using the shooting method because the boundary conditions are defined on both ends of the integration range, generically labeled $\left[x_{1}, x_{2}\right]$. This procedure consists of several steps, which we now outline. Starting at the initial point $x_{1}$ of the integration, suppose that $N$ boundary conditions are to be specified but only $n_{1}$ are provided. This leaves $n_{2} \equiv N-n_{1}$ freely specifiable starting points, from which one can form an $n_{2}$-dimensional vector $\mathbf{V}$, the initial value of which is the first guess for the remaining boundary conditions. We then use the combined boundary conditions to integrate the differential equations and obtain $y\left(x_{2}\right)$ ( $y$ being the 
generic dependent variable label) at the endpoint $x_{2}$ of the integration. Here one forms an $n_{2}$-dimensional discrepancy vector $\mathbf{F}$, which is the difference between the result $y\left(x_{2}\right)$ of the numerical integration and the specified boundary conditions at $x_{2}$. The integration is performed via an adaptive stepsize Runge-Kutta (RK) method with constants provided by Cash and Karp. The solution is then obtained by optimizing the vector $\mathbf{V}$ such that the vector $\mathbf{F}$ converges to zero, by means of the globally-convergent Newton-Raphson method.

As with any numerical procedure, this calculation has an inherent rounding error. There are two main sources for these errors, the RK integration and the Newton-Raphson method.

1. The RK integration routine naturally provides in its result a 5th-order truncation error (called $\frac{1}{15} \Delta$ in NR) on the dependent variable. This truncation error scaled by some combination of the function $y$ and its derivative $d y d x$ (using the terminology of Ref. [36] ) evaluated at the initial point of each step in the integration determines the size $h$ of the next step, but more importantly, drives the desired accuracy of our calculation. In particular, the truncation error is obtained by taking the difference between the result calculated by adaptive stepsize RK (at 5th-order) and the result calculated by classical 4th-order RK. The small dimensionless parameter eps (fractional error) correlated to the truncation error is then defined by $\Delta=\operatorname{eps} \times \operatorname{yscal}(i)$, where $i$ is the index of the particular differential equation among those simultaneously solved that contributes the most to the truncation error (the "worst offender"). We choose the particular recipe for yscal in our calculation to be yscal $(i)=|y(i)|+|h \times d y d x(i)|$ (see NR for other recipes). Lastly, we use eps $=10^{-6}$ in our calculation.

2. The Newton-Raphson method introduces several small parameters to assure convergence:

- The small parameters ALF and TOLMIN are introduced to guarantee the proper convergence of the Newton-Raphson subroutine and therefore affect the final result of the calculation only indirectly. ALF functions as an indicator that the evaluated function has ceased to decrease appreciably, as determined by the Newton-Raphson method. In this case the subroutine backtracks along the Newton direction until an acceptable rate of decrease is obtained. TOLMIN sets the criterion for identifying spurious convergence, in which case the subroutine 
warns of the event and requires the introduction of a new initial point. These parameters are chosen to be ALF $=10^{-4}$ and TOLMIN $=10^{-6}$.

- TOLX sets the convergence criterion for $\delta x$, which represents $\delta \mathbf{V}$ in the shooting method algorithm. Its convergence criterion is set by TOLX $=10^{-7}$.

- $\mathrm{TOLF}$ sets the convergence criterion on $\mathbf{F}$ values; TOLF $=10^{-4}$.

Further details can be found in Ref. [36].

All of these parameters are at least as small as (in most cases, appreciably smaller than) the measured uncertainties of the low-energy QCD observables to which we fit our models. As such, we can safely assume all the errors in our fits come primarily from experimental uncertainties that have been provided in the text, and we do not include further error analysis in our paper. Indeed, the chief figure of merit is the closeness of fit to the central values of certain hadronic quantities, as we now discuss.

The meson masses $m_{\rho}, m_{\pi}$, and $m_{a_{1}}$ are identified as poles in the solutions to Eqs. (6)(10) $[$ see, e.g., Eq. (16)]. One identifies pole masses by noting that the numerical solution near $q^{2} \rightarrow m^{2}$ crosses a singularity and flips sign. Once a mass eigenvalue is identified, its individual bulk-to-boundary propagator is the solution to the relevant wave equation at that value of $q^{2}=m^{2}$, using the shooting method just described. With the normalization for $g_{5}$

chosen, solutions to Eqs. ([6) $-(10)$ at $q^{2}=0$ are used to derive the decay constants $f_{\rho}, f_{\pi}$, and $f_{a_{1}}$ [as in, e.g., Eq. (15)]. The overall quality of our fits is driven chiefly by (the central values of) the three best-measured meson quantities, $m_{\rho}, m_{\pi}$, and $f_{\pi}$. The other parameters in our models such as $A_{c}, B_{c}$, and $\lambda$ are then allowed to float freely.

\section{ACKNOWLEDGMENTS}

This work was supported by the NSF under Grant No. PHY-0456520.

[1] J.M. Maldacena, Adv. Theor. Math. Phys. 2, 231 (1998) [Int. J. Theor. Phys. 38, 1113 (1999)] arXiv:hep-th/9711200; S.S. Gubser, I.R. Klebanov, and A.M. Polyakov, Phys. Lett. B 428, 105 (1998) arXiv:hep-th/9802109; E. Witten, Adv. Theor. Math. Phys. 2, 253 (1998) arXiv:hep-th/9802150. 
[2] J. Polchinski and M.J. Strassler, Phys. Rev. Lett. 88, 031601 (2002) arXiv:hep-th/0109174.

[3] M. Shifman, arXiv:hep-ph/0507246.

[4] A. Karch, E. Katz, D.T. Son, and M.A. Stephanov, Phys. Rev. D 74, 015005 (2006) [arXiv:hep-ph/0602229].

[5] J. Erlich, E. Katz, D.T. Son, and M.A. Stephanov, Phys. Rev. Lett. 95, 261602 (2005) arXiv:hep-ph/0501128.

[6] H. Boschi-Filho and N.R.F. Braga, JHEP 0305, 009 (2003) arXiv:hep-th/0212207].

[7] G.F. de Teramond and S.J. Brodsky, Phys. Rev. Lett. 94, 201601 (2005) arXiv:hep-th/0501022].

[8] N. Evans and A. Tedder, Phys. Lett. B 642, 546 (2006) arXiv:hep-ph/0609112.

[9] D.K. Hong, T. Inami and H.U. Yee, Phys. Lett. B 646, 165 (2007) arXiv:hep-ph/0609270.

[10] P. Colangelo, F. De Fazio, F. Jugeau and S. Nicotri, Phys. Lett. B 652, 73 (2007) arXiv:hep-ph/0703316.

[11] H. Forkel, M. Beyer and T. Frederico, JHEP 0707, 077 (2007) arXiv:0705.1857 (hep-ph)].

[12] L. Da Rold and A. Pomarol, Nucl. Phys. B 721, 79 (2005) arXiv:hep-ph/0501218; JHEP 0601, 157 (2006) arXiv:hep-ph/0510268.

[13] J. Hirn and V. Sanz, JHEP 0512, 030 (2005) arXiv:hep-ph/0507049; J. Hirn, N. Rius and V. Sanz, Phys. Rev. D 73, 085005 (2006) arXiv:hep-ph/0512240.

[14] K. Ghoroku, N. Maru, M. Tachibana and M. Yahiro, Phys. Lett. B 633, 602 (2006) arXiv:hep-ph/0510334.

[15] T. Huang and F. Zuo, arXiv:0708.0936 [hep-ph].

[16] H. Boschi-Filho, N.R.F. Braga and C.N. Ferreira, Phys. Rev. D 73, 106006 (2006) [Erratumibid. D 74, 089903 (2006)] arXiv:hep-th/0512295; O. Andreev and V.I. Zakharov, Phys. Rev. D 74, 025023 (2006) arXiv:hep-ph/0604204]; C.D. White, Phys. Lett. B 652, 79 (2007) arXiv:hep-ph/0701157.

[17] T. Hambye, B. Hassanain, J. March-Russell and M. Schvellinger, Phys. Rev. D 74, 026003 (2006) arXiv:hep-ph/0512089; Phys. Rev. D 76, 125017 (2007) arXiv:hep-ph/0612010.

[18] H.R. Grigoryan and A.V. Radyushkin, Phys. Lett. B 650, 421 (2007) arXiv:hep-ph/0703069.

[19] H.R. Grigoryan and A.V. Radyushkin, Phys. Rev. D 76, 095007 (2007) arXiv:0706.1543 (hep-ph)].

[20] S. Hong, S. Yoon and M.J. Strassler, JHEP 0604, 003 (2006) arXiv:hep-th/0409118. 
[21] A.V. Radyushkin, Phys. Lett. B 642, 459 (2006) arXiv:hep-ph/0605116].

[22] S.J. Brodsky and G.F. de Téramond, arXiv:0707.3859 [hep-ph].

[23] H. R. Grigoryan and A. V. Radyushkin, Phys. Rev. D 76, 115007 (2007) (arXiv:0709.0500 [hep-ph]).

[24] H. J. Kwee and R. F. Lebed, JHEP 0801, 027 (2008) (arXiv:0708.4054 [hep-ph]).

[25] S. Eidelman et al. (PDG), Phys. Lett. B 592, 1 (2004).

[26] J.F. Donoghue, E. Golowich, and B.R. Holstein, Dynamics of the Standard Model (Cambridge University Press, Cambridge 1992)

[27] D.T. Son and M.A. Stephanov, Phys. Rev. D 69, 065020 (2004) arXiv:hep-ph/0304182.

[28] N. Isgur, C. Morningstar, and C. Reader, Phys. Rev. D 39, 1357 (1989).

[29] S.R. Amendolia et al., Phys. Lett. B 138, 454 (1984); Nucl. Phys. B277, 168 (1986).

[30] P. Brauel et al., Phys. Lett. B 69, 253 (1977); P. Brauel et al., Z. Phys. C 3, 101 (1979).

[31] V. Tadevosyan et al. [Jefferson Lab F(pi) Collaboration], Phys. Rev. C 75, 055205 (2007) arXiv:nucl-ex/0607007].

[32] H. Ackermann et al., Nucl. Phys. B137, 294 (1978).

[33] T. Horn et al. [Fpi2 Collaboration], Phys. Rev. Lett. 97, 192001 (2006) arXiv:nucl-ex/0607005].

[34] C.J. Bebek et al., Phys. Rev. D 17, 1693 (1978).

[35] G.P. Lepage and S.J. Brodsky, Phys. Lett. B 87, 359 (1979); A.V. Efremov and A.V. Radyushkin, Theor. Math. Phys. 42, 97 (1980) [Teor. Mat. Fiz. 42, 147 (1980)].

[36] William H. Press, Brian P. Flannery, Saul A. Teukolsky, William T. Vetterling, Numerical Recipes in FORTRAN 77: The Art of Scientific Computing, (Cambridge University Press, 1992).

[37] However, we do not entirely agree with the normalization of their parameters $f_{\pi}$ and $g_{5}$. If we use their values, the numerical agreement is perfect. 